Rev. Elev. Méd, vét. Pays trop., 1971, 24 (2) : $277-86$

\title{
Marquage des glossines par des poudres fluorescentes
}

\author{
par R. TIBAYRENC, J. ITARD et D. CUISANCE (*)
}

\begin{abstract}
RESUME
Dans le but d'étudier, sur le terrain, les lieux de repos nocturnes des glossines, les auteurs ont expérimenté, au laboratoire, l'utilisation pratique d'une méthode de marquage par poudres fluorescentes, non traumatisante pour l'insecte.

Les glossines qui, au moment de l'éclosion, traversent une fine couche de poudre fluorescente, sont décelables, pendant plusieurs semaines, au moyen d'une lampe portative à ultraviolet. Le sac ptilinal, qui conserve, après son invagination, les grains de colorants, permet, après dissection de la tête, de repérer l'insecte jusqu'à la fin de sa vie.
\end{abstract}

\section{INTRODUCTION}

Depuis une dizaine d'années, le marquage des insectes par des substances fluorescentes connaît une grande vogue. Selon l'insecte ou la manière dont la substance fluorescente est appliquée, ces techniques se prêtent à des études écologiques, soit de dispersion (méthode de lâchers-recaptures), soit d'écologie fine (presque d'éthologie), permettant, principalement la nuit, de repérer les sujets marqués et d'observer leur comportement.

Le principe en est simple : l'insecte est, soit poudré à l'aide de poudres fluorescentes, soit revêtu d'une goutte de peinture possédant les mêmes qualités $\left.{ }^{(}\right)$.

Le marquage des Glossines à l'aide de substances fluorescentes sera en particulier utilisé pour l'étude des gîtes de repos nocturnes (resting-sites des Anglo-Saxons). La connaissance parfaite du comportement nocturne des

(1) La fluorescence est la propriété, possédée par certains produjts, d'émettre un rayonnement lumineux de forte intensité sous l'influence d'une lumière excitatrice. Cette émission cesse lorsque l'excitation n’a plus lieu. On différencie en cela la fluorescence de la phosphorescence. La princpale source d'excitation utilisée est la lumière noire (ultraviolets de grande longueur d'onde, environ $3.600 \AA$ ). tsé-tsé revêt en effet un grand intérêt dans l'optique d'une lutte insecticide par épandage sélectif, discriminatoire, qui, tout en économisant le produit employé, diminue les risques de contamination globale du milieu environnant.

La mise en application pratique d'une méthode simple de marquage des glossines nécessitait une étude des conditions d'utilisation de ces substances fluorescentes. Cette étude a été effectuée au laboratoire d'Entomologie de l'I.E.M.V.T. de Maisons-Alfort. Les résultats en sont exposés ici.

\section{METHODES DE POUDRAGE}

\section{a) Les poudres fluorescentes}

Il existe plusieurs marques de poudres fluorescentes. Toutes sont à base de sels de zinc. La principale source de fabrication est américaine, dont les deux marques principales sont Ultra-Violet Products, et Switzer Brothers inc. Pour nos essais de poudrage, nous avons utilisé les poudres Switzer Brothers, distribuées en France par Valentine S.A. ou par la S.F.S. (Société Française de Sérigraphie). Ces sociétés

(*) Institut d'Elevage et de Médecine Vétérinaire des Pays Tropicaux, Laboratoire d'Entomologie. 
ne commercialisent pas les poudres telles quelles, mais les vendent incorporées à des pâtes ou à des vernis destinés à la publicité ou à la signalisation lumineuse. Nous avons pu cependant disposer de quantités assez importantes de quatre coloris = Bow yellow (jaune arc), Fire orange (orange feu), Rocket red (rouge fusée), Signal green (vert signal).

Ces produits se présentent sous forme de poudres extrêmement fines et pulvérulentes (dimension maximale moyenne des particules: $4 \mu$ ). Leur couleur est très vive, même à la lumière du jour ordinaire, et augmente d'intensité, acquérant une grand brillance, sous l'influence excitatrice des U.V. de grande longueur d'onde (lumière noire ou lumière de Wood, de $3.600 \AA$ ).

\section{b) Technique de poudrage des adultes}

D'essais antérieurs effectués par l'un de nous (D.C.), il ressortait que les poudres avaient une action toxique sur les Glossines si elles étaient utilisées en trop fortes quantités.

En conséquence, le poudrage a été effectué de la façon suivante:

Les mouches endormies (par le froid ou le $\mathrm{CO}_{2}$ ) sont déposées dans un récipient en résine synthétique transparente, d'un modèle courant du commerce (destiné à contenir sucre, café, etc.) dont le couvercle comporte une petite trappe. Par cette ouverture, on poudre à l'aide d'un flacon poudreur classique (conditionnement fréquent de poudres pharmaceutiques, sulfamides, talc...). On roule doucement les mouches dans la poudre, en envoyant un ou plusieurs nuages supplémentaires, jusqu'à ce que toutes soient intégralement recouvertes. Ainsi, tous les individus sont complètement poudrés, avec une quantité juste suffisante, puisqu'il ne subsiste pratiquement pas de poudre dans le récipient lui-même.

Les Glossines sont alors replacées dans leur cage (s'il s'agit d'un lot de 20 ou 25) ou dans une grande cage à grosses mailles (quand 100 sujets ou plus ont subi le traitement).

Une fois éveillé, l'insecte cherche à enlever le colorant en pratiquant des mouvements de toilette répétés, se frottant les pattes l'une sur l'autre, sur les ailes, sur le corps.

La poudre qui en tombe salit le tulle des cages, aussi est-il nécessaire de les transférer dans une cage propre 24 heures après. On évite par la même occasion une éventuelle action toxique du surplus de poudre.

Dans une première série d'essais, des mâles de différentes espèces ont été poudrés, pour observer la localisation des poudres sur le tégument et apprécier la durée pratique du marquage.

La toxicité des quatre poudres a été ensuite testée sur les deux sexes de trois espèces: Glossina tachinoides, Glossina austeni et Glossina morsitans. On a constitué, pour ce faire, 24 lots comportant chacun une vingtaine d'individus.

\section{c) Technique de poudrage des Glossines à l'éclosion}

Le simple mélange des pupes et de la poudre destinée au marquage n'est pas une méthode satisfaisante, car beaucoup de mouches à l'émergence restent dans le colorant sans pouvoir en sortir, le produit adhérant sur tout le corps et les empêchant de prendre leur envol normal. En corollaire survient une très forte mortalité les cinq jours suivants.

Pour éviter cet inconvénient, les pupes prêtes à éclore ont été enfouies dans du sable, à un demi-centimètre de profondeur et la surface du sable recouverte d'une fine couche de poudre fluorescente. Ainsi ce n'est qu'en traversant cette couche que les mouches se recouvrent de poudre, pendant un temps assez bref.

Les mouches, une fois leurs ailes déplissées et leurs téguments durcis et foncés, sont transférées de leur cage d'éclosion dans une cage définitive.

\section{LAMPES A RAYONNEMENT ULTRAVIOLET}

Deux sortes de lampes sont nécessaires pour cette étude :

1. une lampe simple, si possible peu coûteuse, destinée à éclairer en lumière noire les Glossines examinées à la loupe binoculaire;

2. une lampe portative projetant des U.V. de grande longueur d'onde à une distance d'au moins deux ou trois mètres, pour une utilisation sur le terrain en brousse. 
a) Lampe d'examen détaillé des Glossines marquées

En général, toutes les lampes à lumière de Wood sont assez coûteuses, car un appareillage d'alimentation particulier doit être interposé entre l'ampoule et la source de courant.

MAZDA a récemment conçu une petite ampoule (TF WN 6), peu commercialisée pour l'instant, d'une puissance de 4 watts, qui se branche directement sur le 220 volt. Son coût est minime (entre 30 et 40 francs).

On obtient une lampe très simple à l'aide d'une douille à vis, d'un morceau de tôle brillante servant de réflecteur et d'un support réglable de paillasse de laboratoire.

\section{b) Lampe portative de brousse}

La firme Ultra-violet Products inc., représentée en France par les Etablissements VILBERT LOURMAT, propose un certain nombre de lampes U.V. fixes et portatives. Les lampes portatives sont très utilisées en minéralogie pour les prospections.

Parmi les différents modèles portatifs (lampe UVL 21 avec ensemble portatif G 203 à piles; lampe M 16 à accus rechargeables; lampe ML 44), le modèle ML 44 a été retenu. Il possède les caractéristiques suivantes :

- induction d'une fluorescence à des distances plus grandes que les appareils plus anciens du même type (4 $\mathrm{m}$ environ),

— batterie (à jeter) d'une durée de 10 heures, incorporée dans l'appareil,

- poignée de manutention peu fatigante pour l'opérateur;

— poids : approximativement $3 \mathrm{~kg}$, batterie comprise,

- finition très soignée.

Son prix en France est d'environ $800 \mathrm{~F}$.

\section{RESULTATS}

\section{a) Poudrage des adultes}

1. Localisation de la poudre sur le tégument

- Durée du marquage

Si tout l'insecte est recouvert de poudre juste après l'opération de poudrage, seuls le thorax et, dans une certaine mesure, la tête (pointe du V de la suture ptilinale, pourtour du scape) restent marqués après 24 heures.

Après quarante-cinq jours, les Glossines poudrées ont été sacrifiées et les dépôts de poudre notés systématiquement.

On note la localisation du colorant principalement aux endroits suivants:

- sur la face dorsale : seul le sillon prescutellaire est très bien marqué; quelques traces subsistent en arrière de la tête, sur le pronotum, et à la base de l'aile;

- sur les faces latérales : le pourtour de la bulla infra alaire et de la zone d'insertion de l'aile sont bien colorés. Il semble que les soies mésopleurales et ptéropleurales maintiennent le colorant et empêchent sa disparition en le protégeant; la région du stigmate prothoracique et les parties supérieures des coxae retiennent également bien ces substances;

-- sur la face ventrale : la poudre, protégée par les saillies des coxae, reste fixée entre celles-ci, permettant de distinguer nettement l'insecte marqué.

\section{Toxicité des poudres fluorescentes}

à l'égard des Glossines

Les lots de Glossines (20 individus) une fois constitués n'ont pas été utilisés immédiatement. Formés à partir de mouches nouvellement écloses, il nous a paru plus rigoureux, pour l'interprétation future des résultats, d'attendre quelques jours avant le poudrage; la mortalité inévitable après l'éclosion ayant éliminé alors les individus fragiles, les lots, bien que parfois inférieurs à 20 individus, étaient par contre bien homogènes.

Les résultats sont consignés dans le tableau I. Ils sont interprétables de la manière suivante :

- pas de différence significative de toxicité entre les quatre poudres pour chaque espèce et chaque sexe;

- pour une même poudre, pas de susceptibilité particulière d'une espèce ou d'un sexe. La mortalité plus forte chez Glossina tachinoides est normale, et constatée également dans les conditions de l'élevage de routine.

La coloration de la poudre n'a pas d'influence sur la longévité, quel que soit l'espèce ou le sexe de la Glossine marquée. 


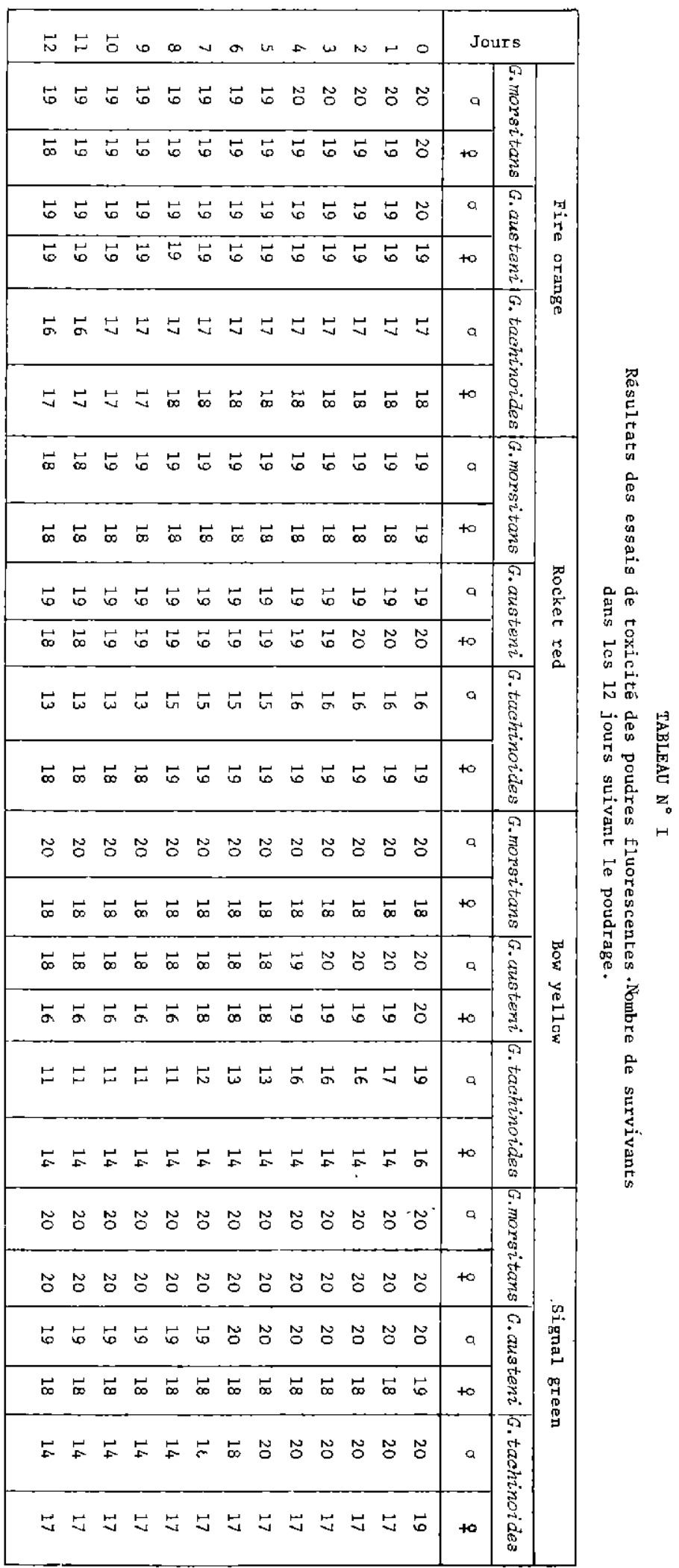




\section{b) Poudrage des Glossines à l'éclosion}

Il n'est pas inutile de rappeler que les Glossines, comme tous les Diptères Cyclorrhaphes Schizophores, provoquent à l'éclosion une rupture circulaire de l'extrémité proximale du puparium à l'aide de leur ptilinum, sac tégumentaire gonflé de lymphe situé au niveau du front. L'imago une fois éclos, le ptilinum s'invagine à l'intérieur de la tête au bout d'un délai plus ou moins long (au moins quelques dizaines de minutes) et n'est plus apparent extérieurement que par sa trace, la suture ptilinale, en forme de lunule à inflexion tournée vers le haut, entourant la base des antennes.

La poudre se localise aux mêmes endroits que lors du poudrage des adultes éclos depuis quelques jours, mais on constate une adhérence plus forte du colorant, qui se fixe en quantité plus importantes. Cela tient sans doute à la légère humidité du tégument à l'éclosion; la poudre " colle" donc davantage.

La localisation la plus intéressante et la plus originale par rapport au marquage des adultes est celle du sac ptilinal. Après quatre semaines, il est possible de repérer sous lampe U.V. les ptilinum marqués. L'insecte décapité, le sac ptilinal est dilacéré sur un papier à chromatographie imprégné de glycérine tamponnée. Le sang parfois contenu dans la tête peut se répandre et masquer partiellement le colorant, ce qui oblige à réaliser une dilacération relativement soignée : on ne peut se contenter d'écraser purement et simplement la tête. La dissection ménagée de la tête sous loupe binoculaire n'est cependant pas une opération compliquée; il est assez facile d'étaler le sac ptilinal et de voir sa paroi interne tapissée de grains fluorescents.

\section{DISCUSSION ET CONCLUSION}

Les méthodes de marquage destinées à l'étude des populations doivent satisfaire aux conditions suivantes:

Elles doivent permettre le traitement rapide d'un grand nombre de Glossines qui seront lâchées ensuite en bloc dans la nature.

Elles ne doivent pas provoquer de mortalité exagérée.

Elles doivent permettre le repérage aisé et durable des Glossines.
En pratique, on devra se plier aux obligations suivantes:

- utiliser des produits non toxiques;

- les appliquer de la manière la moins traumatisante possible, donc réduire au maximum les manipulations.

Toutes ces conditions sont remplies avec le marquage par isotopes radio-actifs. Ces substances sont ingérées par des glossines que l'on fait gorger sur des lapins ayant au préalable reçu, en injection, des substances radio-actives en solution $\left(\mathrm{Fe}^{59}, \mathrm{Zn}^{65}\right)$. Les manipulations des insectes sont réduites au minimum; le marquage dure longtemps et se décèle assez facilement à l'aide d'un compteur portatif type G.M. Cette méthode, par contre, est relativement complexe et coûteuse, car elle implique la manipulation de substances radio-actives et l'utilisation d'un appareillage de détection des rayons $\gamma$.

En ce qui concerne les marquages visuels, l'application de papiers colorés, de paillettes réfléchissantes, de peintures, qui nécessitent pour leur mise en place sur le corps de l'insecte, des manipulations excessives et longues, ne sont qu'un pis-aller.

L'utilisation des poudres fluorescentes paraît par contre souscrire à toutes les conditions requises. Elles permettent de marquer une grande quantité d'individus; elles ne sont pas toxiques; les manipulations sont simples, rapides et peu traumatisantes.

Le poudrage des imagos déjà éclos ne paraît pas offrir de garanties suffisantes quant à la durée et l'intensité du marquage pour une étude des populations de Glossines. Si le colorant est encore décelable au laboratoire au bout de quarante-cinq jours ou même deux mois, il est fort probable qu'il n'en sera pas de même dans la nature, où les mouches risquent de perdre leur poudre, surtout en saison des pluies.

Le marquage des Glossines à l'éclosion, par traversée d'une mince couche de poudre fluorescente, devrait donner de bons résultats en pratique, en raison de l'humidité de leur tégument. La poudre reste de toute évidence pour la vie entière dans le sac ptilinal de l'individu.

Cette méthode a été utilisée avec succès dans des études de dispersion de Diptères d'intérêt agricole, à Hawaï et Capri notamment.

Il eut été intéressant de tester la méthode utilisée par certains auteurs américains (GAI- 
NES, ROTH et PLAPP), qui " givrent " avant poudrage les Diptères dont ils étudient l'écologie (Stomoxys calcitrans, Haematobia irritans, Musca domestica), à l'aide d'une substance nommée "Plyac». Il nous fut malheureusement impossible d'identifier ce produit, qui n'est désigné que par son appellation commerciale.

Le simple poudrage offre cependant un grand intérêt, en raison de sa simplicité, pour l'étude des gîtes de repos nocturne. Un des procédés d'utilisation pourrait consister à introduire, après marquage, dans une grande cage en toile métallique de plusieurs mètres cubes posée autour d'un échantillon représentatif de la végétation de la galerie étudiée, des glossines d'élevage ou des glossines sauvages capturées sur le lieu même de l'étude, qui seraient ensuite décelées la nuit à l'aide du projecteur portatif à U.V. ML 44. Ce protocole, facile à mettre en auvre, devrait permettre de noter d'une manière statistique et rigoureuse la répartition nocturne des Glossines (troncs d'arbres, branches selon leur diamètre et leur orientation, feuilles, creux de racines, etc.).

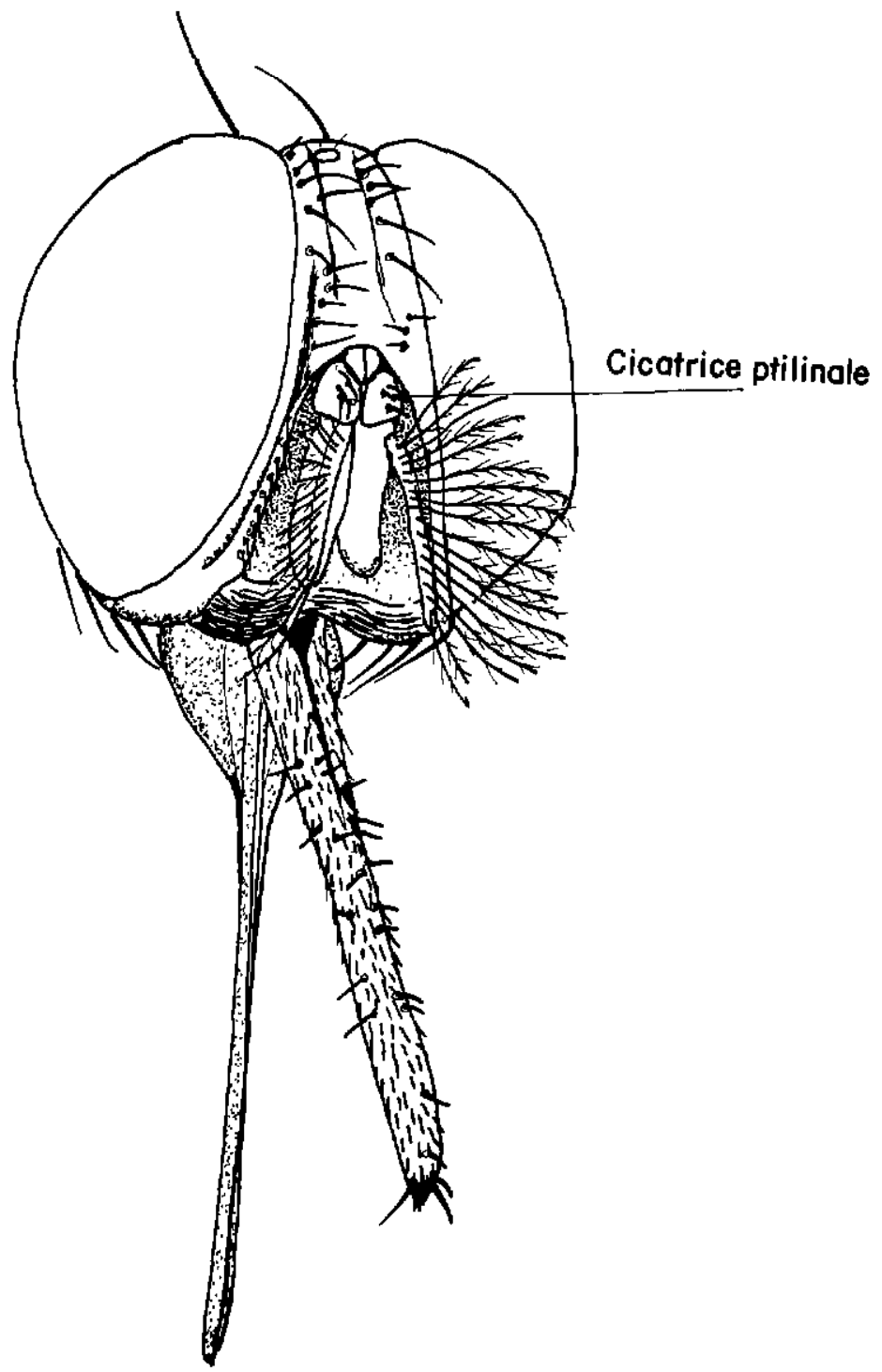

Fig. 1.

Glossina austeni - tête vue de trois-quart avant. 


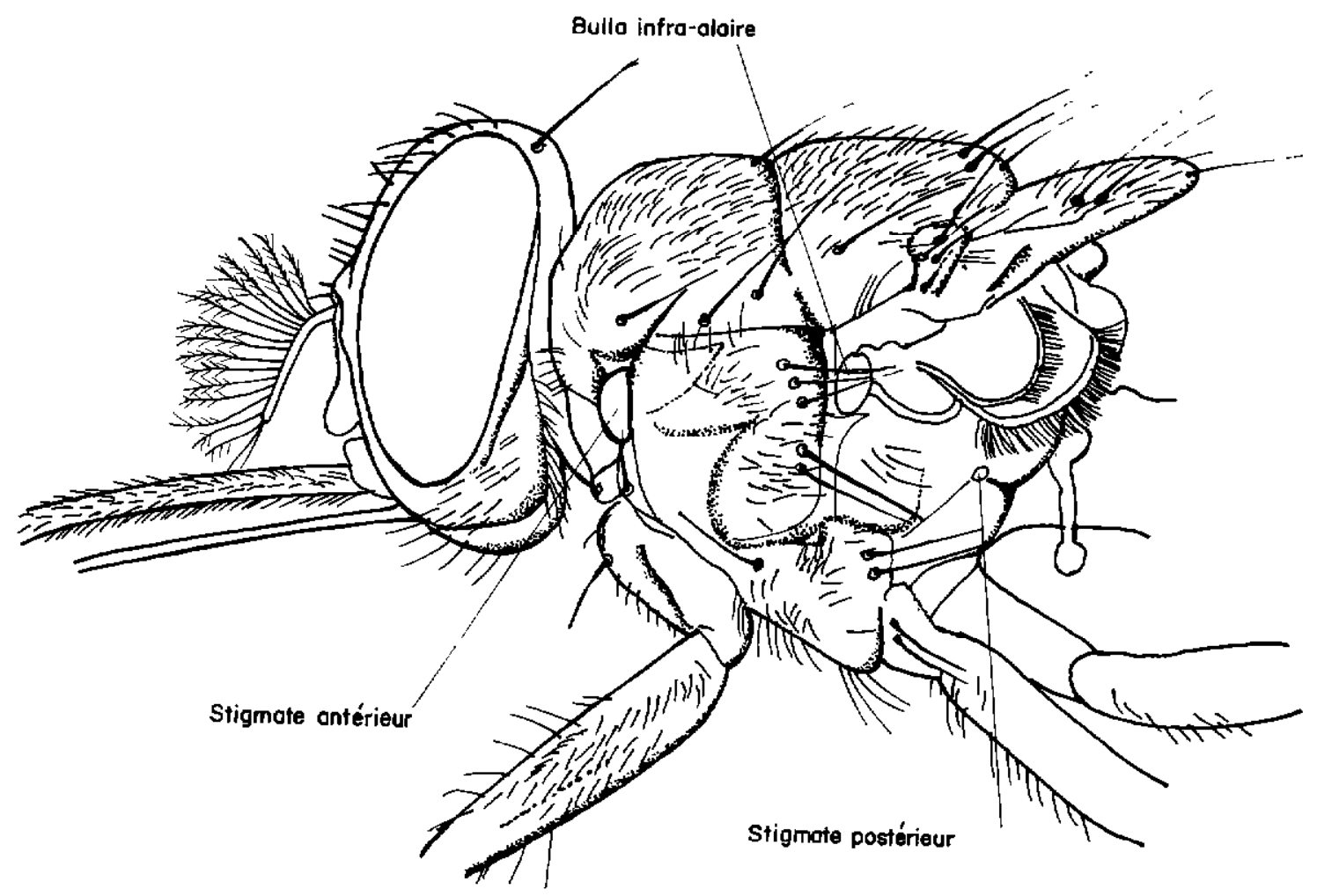

F1g. 2.

Glossina austeni - thorax; vue latérale. 


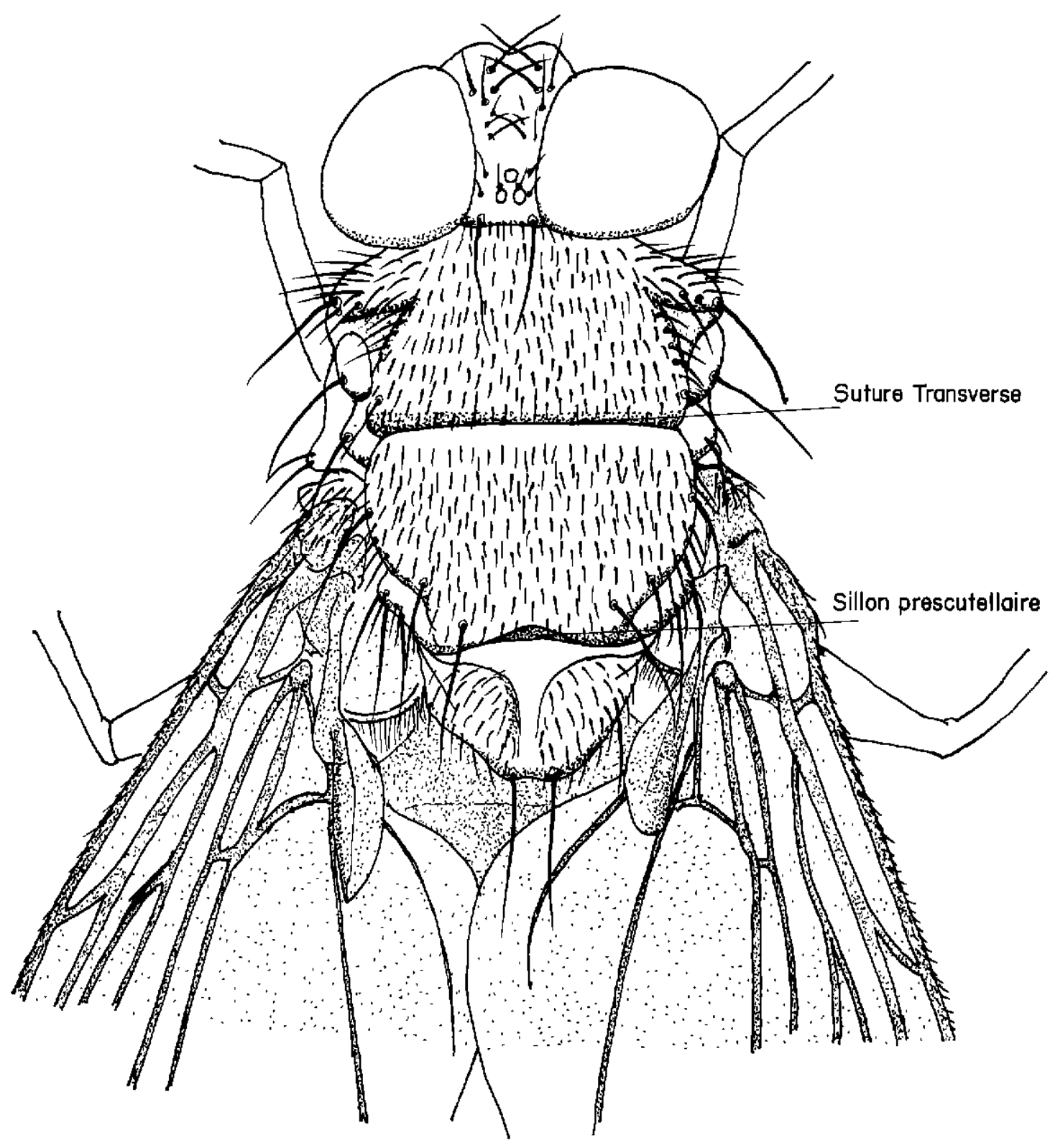

Fig. 3.

Glossina austeni - thorax; vue supérieure. 


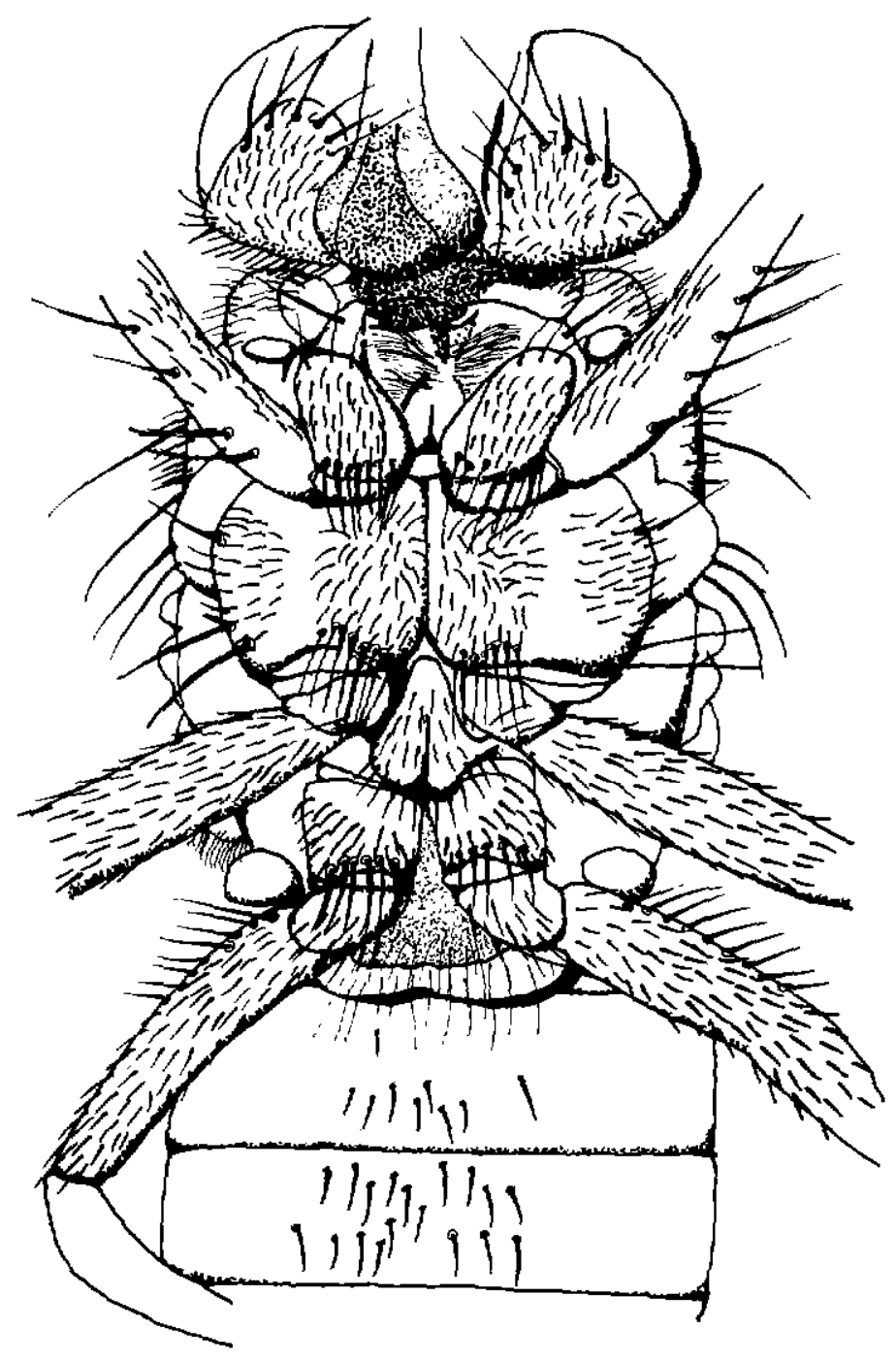

Fig. 4.

Glossina austeni - thorax, vue inférieure. 


\section{SUMMARY}

\section{Marking of tse-tse flies by fluorescent powders}

In order to study in the field the resting sites of tse-tse flies, the authors have experimented in the laboratory the practical application of a marking method using fluorescent powders, not harmful to the insect.

During emergence, the flies cross a thin layer of fluorescent powder and can be identified during several weeks with a portable ultra-violet lamp. The ptilinum retains the dye particles after its invagination and allows identification of the insect during his whole life, after dissection of the head.

\section{RESUMEN}

\section{Marca de las glosinas mediante polvos fluorescentes}

Con el objeto de estudiar, sobre terreno, los sitios de descanso nocturno de las glosinas, los autores experimentaron, en laboratorio, la utjlización práctica de un método de marca mediante polvos fluorescentes, no peligrosos para el insecto.

Se pueden descubrir, durante varias semanas mediante una lámpara portatil con ultravioleta, las glosinas que, al momento de la salida, atravesan una capa fina de polvo fluorescente. El saco ptilinal que conserva, después de su invaginación, los granos de colorantes, permite, después de la disección de la cabeza, señalar el insecto hasta el fín de su vida.

\section{BIBLIOGRAPHE}

ADKINS $\mathbf{J}_{\Gamma}$ (T. R.), « A foot controlled device using carbon dioxide for the anesthetization of insects ", J. econ. Ent., 1968, 61 (1): 340-41.

BAILEY (S. F.), ELIASON (D. A.) et ILTIS (W. C.), «Some marking and recovery techniques in Culex tarsalis Coq, flight studies », Mosquito news, 1962, 22: $1-10$.

BRUES (C. T.), «Fluorescent staining of insect tissues », Science, 1944, $100(260)$ : 554-55.

BULOW (F.J.) et HUGGINS (D. G.), « Mark and recapture methods for studying domestic cockroach populations 》, Proc. Iowa Acad. Sci., 1968, 75: $447-56$.

CUISANCE (D.) et ITARD (J.), « Technique de marquage des Glossines au moyen de radio-isotopes $»$, Symposium on the sterility principle for insect control or eradication I.A.E.A./F.A.O. - Athènes, 14-18 septembre 1970.

DEAN (G. J. W.), CLEMENTS (S. A.) et PAGET (J.), «Observations on sex attraction and mating behaviour of the tsetse fly, Glosina morsitans orientalis Vanderplank », Bull. Ent. Res., 1968, 59: 355-65.

«Dye marked flies easily identified », USDA Agric. Res., 1965, 13 (11) : 15-16.

EDDY (G.W.), ROTH (A. R.) et PLAPP (F.W.), «Studies on the flights habits of some marked insects $», J$. econ. entomol., 1962, 55 (5) : 603-07.

GANGWERE (S. K.), CHAVIN (W.) et EVANS (F. C.), "Methods of marking insects with special references to Orthoptera (s.1.) 》, Ann. ent. soc. Amer., 1964, 57 (6) : 662-69.

HARRIS (R. L.) et FRAZAR (E. D.), «A device for immobilizing insects with cooled air $*, J$. econ. Ent., 1968, 61 (6) : 1755-56.

HARRIS (R. L.), HOFFMANN (R. A.) et FRAZAR (E. D.), \& Chilling us other methods of immobilizing flies », J. econ. Ent., 1965, 58 (2) : 379-80.
HOLBROCK (F.R.), STEINER (J.F.) et FUJIMOTO (M.S.), « Mating competitiveness of mediterranean fruit flies marked with fluorescent powders », J. econ. Ent., 1970, 63 (2) : 454-55.

KNUDSEN (A. B.) et REES (Don M.), « Methods used in Utah for sampling tabanid populations", Mosq. news, 1968, 28 (3) : 356-62.

MEDLEY (J.G.) et AHRENS (E. H.), « Fluorescent dyes for marking and recovering fowl ticks in poultry houses treated with insecticides $" J$. econ. Ent., 1968, 61 (1) : 81-84.

MUSGRAVE (A.J.), "The use of fluorescent materials for marking and detecting insects », Canadian Ent., 1949, 81, 173.

PATTON (R.L.), EDWARDS (L. J.) et GILMORE (S. K.), «Delivery safe levels of $\mathrm{CO}_{2}$ for insects anesthesia ", Ann. ent. Soc. Amer., 1968, 61 (4): 1046-47.

POLIVKA (J. B.), «The use of fluorescent pigments in a study of the flight of the Japanese Beetle $"$, J. econ. Ent., 1949, 42: 818-21.

TAFT (H. M.) et AGEE (H. P.), «A marking and recovery method for use in Boll weevil movement studies », J. econ. Ent., 1962. 55 (6) : 1018-19.

TURNER Jr (E.C.) et GERHARDT (R. R.), «A materials for rapid marking of face flies for dispersal studies », J. econ. Ent., 1965, 58 (3) : 584-85.

VAIL (P. V.), HOWLAND (A. F.) et HENNEBERRY (T. J.), « Fluorescent dyes for mating and recovery studies with cabbage looper moths $\gg, J$. econ. Ent., $1966, \mathbf{5 9}, 1093-97$.

WOLF (W. W.), KILLOUGH (R. A.) et HARTSOCK (J.G.), «Small equipment for immobilizing flies with cool air », J. econ. Ent., 1967, 60 (1) : 303-04.

WOOD (F. G.), «A new method of pointing insects", J. Kansas ent. soc., 1965, 38 (2) : 203-04.

WOODRINC (J. P.) et CUTCHER (J. J.), « Vital dye marking of tyroglyphid mites ", Ann. ent. soc. Amer., 1968, 48 (4) : 1031-32. 